\title{
Mathematical modelling of conjugate formation by cytotoxic lymphocytes and tumour cells
}

\author{
J. Waniewski ${ }^{\text {I) }}$, A. K. Palucka ${ }^{2)}$ and A. Porwit ${ }^{3)}$
}

1) Baxter Novum, Department of Clinical Sciences, Huddinge University Hospital, Huddinge, Sweden and Institute of Biocybernetics and Biomedical Engineering, Warsaw, Poland. 2) Department of Haematology, Karolinska Hospital, Hospital, Stockholm, Sweden.

3) Department of Pathology, Karolinska Hospital, Stockholm, Sweden

Jacek Waniewski, Department of Renal Medicine K56, Huddinge University Hospital, S-141 86 Huddinge, Sweden.

Tel: 46-8-746 3292. Fax: 46-8-746 3290 .

e-mail: jacek@kfc.hs.sll.se

\begin{abstract}
Kinetics of conjugate formation between cytotoxic lymphocytes and tumour cells is described using the enzyme kinetics model and adapted for the evaluation of in vitro measurements by double fluorescence flow cytometry. The parameters of the model were estimated for two effector-target systems. A previously reported model of the first order kinetics is shown to be an approximation for the presented model. A parameter, derived from the model and called the strength of binding, is proposed for a simple and objective evaluation of the experiments.
\end{abstract}

\section{Keywords}

Conjugate formation, enzyme kinetics, first order kinetics, flow cytofluorometry 


\section{INTRODUCTION}

The effect of cytotoxic lymphocytes on tumour cells includes usually three steps: 1) binding of the lymphocyte to a target and formation of a conjugate, 2) cytotoxic "hit" which causes a damage of the target membrane and invokes lethal processes inside the cell, and 3) dissolution of the conjugate and target's death. The formation of conjugates can be investigated in vitro using tumour cell lines or blasts from patients with leukaemia. It is possible to stimulate in vitro patient's cytotoxic lymphocytes with interleukin 2 (lymphokine activated killers, LAK, and adherent LAK, ALAK) and applied the stimulated cells in therapy of leukaemia. Therefore, a method for analysis of the lymphocyte cytotoxic effects at each stage of the interaction with targets is necessary. Whereas the overall cytotoxic effect is routinely evaluated by ${ }^{51} \mathrm{Cr}$ release assay (Thoma et al., 1978), various measures of the effectiveness of the conjugate formation have been previously proposed. Some of them are based on the dose-response curve analysis (Garcia-Penarnubia et al., 1989b). In contrast, we investigated in detail the kinetics of conjugate formation and applied a mathematical model based on an enzyme kinetics description (Waniewski et al., 1993).

\section{EXPERIMENTAL METHODS}

A short description of experimental methods applied in the investigations of conjugate formation between cells is necessary to understand the details of the presented model (Waniewski et al., 1993). Each population of the interacting cells is labelled with a specific fluorescent marker, e.g. effectors with a red fluorescence and targets with a green one. Some of cells are not labelled, as they do not bind specific markers (dark or negative cells). The labelled populations (usually with a small fraction of dark cells) are mixed so as to get a definite effector to target ratio (which should be about 1:1 for the experiments described by the model), spun for a short time in a centrifuge, so that they form a pellet, and incubated for various periods of time. After the incubation, the cells are resuspended and kept at $0^{\circ} \mathrm{C}$ to prevent further conjugation. The flow cytometric measurements (e.g. with FACScan) provide the numbers of characteristics counts of each population, e.g., single red fluorescence - free effectors, single green fluorescence - targets, double (red and green) fluorescence - conjugates, dark particles not stained cells, per a definite number of total counts (usually 5 - 10 thousand).

\section{MODEL}

The model has been derived for the conditions in which the conjugates include only one effector cell (cytotoxic lymphocyte) and one target cell (tumour cell); this means that the initial ratio of target to effector cells should be close to 1 (Garcia-Penarrubia et al., 1989a, Waniewski et al., 1993). Three variables describe the interacting cells: the concentration of free, i.e. not bound in conjugates, effector cells, $E_{\mathrm{F}}$, the concentration of free target cells, $T_{\mathrm{F}}$, and the concentration of conjugates of effector and target cells, $C$. A number of other (dark) cells, with concentration $D$, is also present in the experiments but not involved in the cellular interactions. 
The interactions between target and effector cells are described by the enzyme kinetics model (Murray, 1989), with additional possibility of the death of free effectors and free targets:

$$
\begin{aligned}
& \dot{E}_{\mathrm{F}}=-\mathrm{k}_{+} E_{\mathrm{F}} T_{\mathrm{F}}+\mathrm{k}_{-} C-\mathrm{k}_{\mathrm{E}} E_{\mathrm{F}}, \\
& T_{\mathrm{F}}=-\mathrm{k}_{+} E_{\mathrm{F}} T_{\mathrm{F}}+\mathrm{k}_{-} C-\mathrm{k}_{\mathrm{T}} T_{\mathrm{F}}, \\
& \dot{C}=\mathrm{k}_{+} E_{\mathrm{F}} T_{\mathrm{F}}-\mathrm{k}_{-} C,
\end{aligned}
$$

where $k_{+}$is the association rate constant, $k_{-}$is the dissociation rate constant, $k_{E}$ and $k_{T}$ are the disappearance rate constants for free effectors and free targets, respectively. The population of dark cells may increase because of darkening of labelled $T_{\mathrm{F}}$ and $E_{\mathrm{F}}$ (which is interpreted as the death of these cells) and may decrease because of the disintegration of the dark cells:

$$
\dot{D}=\mathrm{k}_{\mathrm{E}} E_{\mathrm{F}}+\mathrm{k}_{\mathrm{T}} T_{\mathrm{F}}-\mathrm{k}_{\mathrm{D}} D
$$

The total concentration of cells, $N$, given as $N=E_{\mathrm{F}}+T_{\mathrm{F}}+2 C+D$, changes with time according to the following law, which is the consequence of equations (1) - (4):

$$
\dot{N}=-\mathrm{k}_{\mathrm{D}} D
$$

whereas the total amount of "particles" (or "events") counted in flow cytometry, $M$, given as $M=E_{\mathrm{F}}+T_{\mathrm{F}}+C+D$, changes as:

$$
\dot{M}=\mathrm{k}_{\mathrm{E}} E_{\mathrm{F}} T_{\mathrm{F}}-\mathrm{k}_{-} C-\mathrm{k}_{\mathrm{D}} D,
$$

If the time of the experiment is short enough to make the lysis of cells negligible (less than 30 $\min$ ), then the total amount of cells, $N$, could be assumed to be approximately constant. In contrast, the amount of "particles", $M$, depends on the stage of the process of conjugate formation, see equation 6 .

In the typical experimental conditions it is not possible to count whole involved cell populations, which usually include about $10^{6}$ cells. Therefore, a sample from the whole population is investigated and the fraction of a particular subpopulation in the sample is considered to be an estimate of the fraction of this subpopulation in the whole population. The model has been adapted to this situation and new variables have been introduced: for free effectors $\varepsilon_{\mathrm{F}}=E_{\mathrm{F}} / N$, for free targets $\tau_{\mathrm{F}}=T_{\mathrm{F}} / N$, for conjugates $\gamma=C / N$, and for dark cells, $\delta=$ $D / N$ (however, these fractions are not directly measured with FACScan and have to be calculated from the fractions of each population measured per number of counted "events", see Perez et al. (1985), Segal et al. (1984), Waniewski et al. (1993)). Because $\varepsilon_{\mathrm{F}}+\tau_{\mathrm{F}}+2 \gamma+\delta=1$, only three equations for subpopulation fractions may be considered:

$$
\begin{aligned}
& \dot{\varepsilon}_{\mathrm{F}}=-\mathrm{k}_{+} N \varepsilon_{\mathrm{F}} \tau_{\mathrm{F}}+\mathrm{k}_{-} \gamma-\mathrm{k}_{\mathrm{E}} \varepsilon_{\mathrm{F}}+\mathrm{k}_{\mathrm{D}} \delta \varepsilon_{\mathrm{F}}, \\
& \dot{\tau}_{\mathrm{F}}=-\mathrm{k}_{+} N \varepsilon_{\mathrm{F}} \tau_{\mathrm{F}}+\mathrm{k}_{-} \gamma-\mathrm{k}_{\mathrm{T}} \tau_{\mathrm{F}}+\mathrm{k}_{\mathrm{D}} \delta \tau_{\mathrm{F}}, \\
& \dot{\gamma}=\mathrm{k}_{+} N \varepsilon_{\mathrm{F}} \tau_{\mathrm{F}}-\mathrm{k}_{-} \gamma+\mathrm{k}_{\mathrm{D}} \delta \gamma .
\end{aligned}
$$


The total concentration of cells, $N$, is usually relatively constant during short experiments aimed on the investigations of conjugate formation, and equal to its initial value, $\mathrm{N}_{0}$. This means that $k_{D} \delta<<1$, c.f. equation (5), and therefore the terms with $k_{D} \delta$ in equations (7) - (9) may be considered negligible. The final form of the model is as follows:

$$
\begin{aligned}
& \dot{\varepsilon}_{\mathrm{F}}=-\mathrm{k}_{+} \mathrm{N}_{0} \varepsilon_{\mathrm{F}} \tau_{\mathrm{F}}+\mathrm{k}_{-} \gamma-\mathrm{k}_{\mathrm{E}} \varepsilon_{\mathrm{F}}, \\
& \dot{\tau}_{\mathrm{F}}=-\mathrm{k}_{+} \mathrm{N}_{0} \varepsilon_{\mathrm{F}} \tau_{\mathrm{F}}+\mathrm{k}_{-} \gamma-\mathrm{k}_{\mathrm{T}} \tau_{\mathrm{F}}, \\
& \dot{\gamma}_{\mathrm{F}}=\mathrm{k}_{+} \mathrm{N}_{0} \varepsilon_{\mathrm{F}} \tau_{\mathrm{F}}-\mathrm{k}_{-} \gamma .
\end{aligned}
$$

The concentration $\mathrm{N}_{0}$ is the concentration of all cells in the pellet formed during centrifugation, and, as the volume of the pellet is not usually measured, $\mathrm{N}_{0}$ is not known. Therefore, the lumped parameter $\mathrm{k}_{+} \mathrm{N}_{0}$, called the binding capacity constant, is estimated.

\section{ANALYSIS OF THE MODEL}

\section{Strength of binding}

Based on the model, a parameter $\mathrm{Sb}$, defined as $\mathrm{Sb}=\mathrm{k}_{+} \mathrm{N}_{0} / \mathrm{k}$. and called the strength of binding, has been proposed for the evaluation of the experimental results (Palucka et al., 1995). Note that $\mathrm{Sb}$ is related to the equilibrium constant, $\mathrm{K}=\mathrm{k} / \mathrm{k}$. This parameter may be evaluated using only one measurement of the cell population fractions, $\gamma_{\mathrm{S}}, \varepsilon_{\mathrm{FS}}$ and $\tau_{\mathrm{FS}}$, within the period of the steady state of the system, as follows from equation (12) for $\dot{\gamma}_{\mathrm{F}}=0$ :

$\mathrm{Sb}=\frac{\gamma_{S}}{\varepsilon_{F S} \tau_{F S}}$

The steady state values $\gamma_{\mathrm{S}}, \varepsilon_{\mathrm{FS}}$ and $\tau_{\mathrm{FS}}$ depend only on one parameter, Sb, which characterises the interaction between effectors and targets, and two additional parameters, the fraction of all effectors, $\varepsilon_{W}=\varepsilon_{F}+\gamma$, and the fraction of all targets, $\tau_{W}=\tau_{F}+\gamma$, which characterise experimental conditions and are relatively constant during the initial phase of conjugate formation (Waniewski et al., 1993, Palucka et al., 1995). Thus, the definition of $\mathrm{Sb}$ and equation (13) yield:

$\gamma_{S}=\frac{1+\operatorname{Sb}\left(\varepsilon_{W}+\tau_{W}\right)-\sqrt{\operatorname{Sb}^{2}\left(\varepsilon_{W}-\tau_{W}\right)^{2}+1+2 \operatorname{Sb}\left(\varepsilon_{W}+\tau_{W}\right)}}{2 S b}$

The equations for $\varepsilon_{F S}$ and $\tau_{F S}$ can be easily formulated as $\varepsilon_{F S}=\varepsilon_{W}-\gamma_{S}$ and $\tau_{F S}=\tau_{W}-\gamma_{S}$, respectively, together with equation (14) for $\gamma_{\mathrm{s}}$. It should be noted however, that time which the system needs to reach the steady state depends on both kinetic parameters, $k_{+} N_{0}$ and $k$, in contrast to the values of variables at the steady state which depend only on the ratio of these kinetic parameters, i.e. on $\mathrm{Sb}$. 


\section{Approximation with first order kinetics}

Equations (10) - (12) may be written in another form using the relationships $\varepsilon_{\mathrm{F}}=\varepsilon_{\mathrm{W}}-\gamma$ and $\tau_{\mathrm{F}}$ $=\tau_{\mathrm{W}}-\gamma$ as follows:

$\dot{\varepsilon}_{\mathrm{F}}=\mathrm{a}_{\mathrm{E}}-\mathrm{b}_{\mathrm{E}} \varepsilon_{\mathrm{F}}$

$\dot{\tau}_{\mathrm{F}}=\mathrm{a}_{\mathrm{T}}-\mathrm{b}_{\mathrm{T}} \tau_{\mathrm{F}}$,

$\dot{\gamma}=\mathrm{a}_{\mathrm{C}}-\mathrm{b}_{\mathrm{C}} \varepsilon_{\mathrm{C}}$,

where

$$
\begin{aligned}
& a_{E}=k_{-} \varepsilon_{W}, \quad b_{E}=k_{+} N_{0} \tau_{W}+k_{-}-k_{E}-k_{+} N_{0} \gamma, \\
& a_{T}=k_{-} \tau_{W}, \quad b_{T}=k_{+} N_{0} \varepsilon_{W}+k_{-}-k_{T}-k_{+} N_{0} \gamma, \\
& a_{C}=k_{-} \varepsilon_{W} \tau_{W}, \quad b_{C}=k_{+} N_{0}\left(\varepsilon_{W}+\tau_{W}\right)+k_{-}+k_{+} N_{0} \gamma .
\end{aligned}
$$

The parameters "a" are relatively constant if $\varepsilon_{\mathrm{W}}$ and $\tau_{\mathrm{W}}$ are relatively constant, see Waniewski et al. (1993), and the parameters " $b$ " may be considered approximately constant if $\gamma$ (which is usually lower then $\varepsilon_{\mathrm{W}}$ and $\tau_{\mathrm{W}}$ ) is approximated by its average value. Therefore, the kinetic of the process of conjugate formation may be approximately described by the first order model, as proposed by Segal et al. (1984) and Perez et al. (1985). Note however, that this kind of kinetic description does not provide direct information about cellular interactions, and depends on the assumption that changes of $\gamma, \varepsilon_{W}$ and $\tau_{W}$ over time may be neglected for the evaluation of the kinetic coefficients.

\section{Parameter estimation}

The parameters of the model $k_{+} N_{0}, k_{-}, k_{E}$, and $k_{T}$ may be estimated with a simplified method of one- and two-parameter linear regressions using the following equations:

$$
\begin{aligned}
& \varepsilon_{\mathrm{W}}(\mathrm{t})=\varepsilon_{\mathrm{W}}(0)-\mathrm{k}_{\mathrm{E}} \int_{0}^{\mathrm{t}} \varepsilon_{\mathrm{F}}(\mathrm{s}) \mathrm{ds}, \\
& \tau_{\mathrm{W}}(\mathrm{t})=\tau_{\mathrm{W}}(0)-\mathrm{k}_{\mathrm{T}} \int_{0}^{\mathrm{t}} \tau_{\mathrm{F}}(\mathrm{s}) \mathrm{ds} \\
& \gamma(t)=\gamma(0)+k_{+} N_{0} \int_{0}^{t} \varepsilon_{F}(s) \tau_{F}(s) d s-k_{-} \int_{0}^{t} \gamma(s) d s,
\end{aligned}
$$

This method of parameter estimation has been found quite precise (Waniewski et al., 1993). Alternatively, the values of the transport parameters estimated with equations (21) - (23) may be used as the initial parameter values for a nonlinear regression applied to equations (10) (12). 


\section{RESULTS}

The model was applied for the evaluation of the experiments with lymphokine activated killers (LAK) interacting with K562 or Daudi cell lines (Waniewski et al., 1993). The fraction of the cell populations were examined at $0,5,10,15,20,25$ and 30 min from the beginning of the incubation using dual parameter flow cytometry (FACScan). The number of conjugates increased for the initial 15-20 min and then remained stable till the end of the experiment. The estimated parameters as well as the strength of binding, $\mathrm{Sb}$, are presented in Table 1.

Table 1. Model parameters estimated for LAK cells incubated with K562 or Daudi targets

\begin{tabular}{lcc}
\hline Parameters & $\begin{array}{c}\mathrm{K} 562-\mathrm{LAK} \\
\operatorname{mean} \pm \mathrm{SD}(\mathrm{n}=8)\end{array}$ & $\begin{array}{c}\text { Daudi-LAK } \\
\text { mean } \pm \mathrm{SD}(\mathrm{n}=4)\end{array}$ \\
\hline $\mathrm{k}_{\mathrm{+}} \mathrm{N}_{\mathbf{0}}[1 / \mathrm{min}]$ & $0.196 \pm 0.049$ & $0.101 \pm 0.020^{* *}$ \\
$\mathrm{k}_{-}[1 / \mathrm{min}]$ & $0.053 \pm 0.025$ & $0.125 \pm 0.045^{* *}$ \\
$\mathrm{k}_{\mathrm{E}}[1 / \mathrm{min}]$ & $0.010 \pm 0.006$ & $-0.000 \pm 0.003^{*}$ \\
$\mathrm{k}_{\mathrm{T}}[1 / \mathrm{min}]$ & $0.016 \pm 0.012$ & $-0.001 \pm 0.005^{*}$ \\
$\mathrm{Sb}^{\mathrm{a}}$ & $3.8 \pm 1.1$ & $1.0 \pm 0.5^{* *}$ \\
\hline
\end{tabular}

à calculated using equation (13)

*,** denote statistical significance ( $\mathrm{P}<0.05$ and $\mathrm{P}<0.01$, respectively), according to Student's $\mathrm{t}$ test, for the difference between the K562-LAK and Daudi-LAK systems.

The model yielded the life times, calculated as the inverse of $k$, for the investigated conjugates, which were on average 8 min for Daudi - LAK and 19 min for K562 - LAK. A good correlation between the values of $\mathrm{Sb}$ calculated using its definition and the estimated values of $\mathrm{k}_{4} \mathrm{~N}_{0}$ and $\mathrm{k}_{\text {- }}$ and the values of $\mathrm{Sb}$ calculated using equation (13) was found (Palucka et al., 1995).

For the evaluation of Sb using equation (13) an experiment may be simpler and less laborious than the study of kinetics of conjugate formation, and therefore $\mathrm{Sb}$ may be easily applied for investigation of different cell systems. In particular, interactions between peripheral blood lymphocytes, LAK, ALAK and various subpopulations of LAK as effectors and cell lines of lymphoid and myeloid origin as well as blast from patients with acute myeloid leukaemia as targets were described using Sb as a characteristic parameter (Palucka et al,, 1995).

\section{SUMMARY}

The model provides a useful and precise description of the dynamic phenomenon of the conjugate formation by cytotoxic lymphocytes and tumour cells, and may be applied for evaluation and comparison of the interactions in various cell systems, including blasts from 
patients with leukaemia. An extension of the model for the description of formation of conjugates with more than two cells and the investigation of such conjugates with FACScan are possible, but have not been performed yet.

\section{REFERENCES}

Garcia-Penarrubia, P., Koster, F.T., Bankhurst, A.D. (1989a) Model for population distributions of lymphocyte-target cell conjugates. $J$ Theor Biol, 138, 77-92.

Garcia-Penarrubia, P., Koster, F.T., Bankhurst, A.D. (1989b) The maximum conjugate frequency $\left(\alpha_{\max }\right)$ characterizes killer cell populations. J Immunol Methods, 118, 199-208.

Murray, J.D. (1989) Mathematical Biology. Springer, Berlin.

Palucka, A.K., Waniewski, J. and Porwit, A. (1995) Strength of binding between leukemic blasts and cytotoxic lymphocytes. Cytometry, submitted.

Perez, P., Bluestone, J.A., Stephany, D.A. and Segal DM. (1985) Quantitative measurements of the specificity and kinetics of conjugate formation between cloned cytotoxic $T$ lymphocyte and splenic target cells by dual parameters flow cytometry. J Immunol, 134, 478-85.

Segal, D.M. and Stephany, D.A. (1984) The measurement of specific cell: cell interactions by dual-parameter flow cytometry. Cytometry, 5, 169-81.

Thoma, J.A., Touton, M.H. and Clark, W.R. (1978) Interpretation of ${ }^{51} \mathrm{Cr}$-release data: a kinetic analysis. $J$ Immunol, 120, 991-7.

Waniewski, J., Palucka, A.K. and Porwit, A. (1993) Kinetic analysis of cytotoxic lymphocyte target cell interaction as quantified by dual parameter flow cytometry. Cytometry, 14, 393400 . 